\title{
Active asteroids: mystery in the Main Belt
}

\author{
Henry H. Hsieh and David Jewitt \\ Institute for Astronomy, University of Hawaii, 2680 Woodlawn Drive, \\ Honolulu, HI, 96822, USA \\ Emails: hsieh@ifa.hawaii.edu and jewitt@ifa.hawaii.edu
}

\begin{abstract}
Classically, comets from the outer solar system (beyond the orbit of Neptune), are expected to be icy, and thus active near the Sun, while asteroids in the inner solar system (interior to the orbit of Jupiter) are expected to be relatively ice-deficient, and thus inert. Studies of anomalous objects, most recently 133P/Elst-Pizarro, challenge this classical picture, however, and suggest that either (1) subsurface ice can in fact be preserved over billions of years in small bodies in the inner solar system but still be close enough to the surface to be excavated by an impact by another body, or (2) non-gravitational dynamical evolution (primarily driven by asymmetrical outgassing) of icy bodies from the outer solar system can drive these cometary bodies onto thoroughly asteroid-like orbits, erasing all dynamical signs of their trans-Neptunian origins in the process. The question thus boils down to whether occasionally sublimating icy bodies on stable asteroid-like orbits in the inner solar system, particularly in the main asteroid belt, may in fact be native to the region or whether they must necessarily be recent arrivals.
\end{abstract}

Keywords. comets: general comets: individual (133P/Elst-Pizarro), minor planets, asteroids

\section{Introduction}

Classically, comets and asteroids are considered distinct on four levels: observational, physical, dynamical, and evolutionary. The observational distinction is the simplest: comets are "fuzzy", displaying comae and tails, while asteroids are not. This arises from an assumed physical distinction: comets contain significant quantities of volatile material, i.e. ices, which sublimate when heated by the Sun, while asteroids do not. This physical distinction is in turn a consequence of dynamics: comets occupy highly eccentric orbits, spending large amounts of time in the cold outer solar system where their primordial volatile supplies are preserved against depletion by solar heating, while most asteroids occupy more circular orbits in close proximity to the Sun and thus receive much more solar heating over their lifetimes, making the survival of primordial ices (if any) correspondingly more difficult. This dynamical distinction is frequently defined by the Tisserand parameter, $T_{J}$, an invariant of motion in the restricted three-body problem. Objects with $T_{J}<3$ are dynamically coupled to Jupiter and are considered cometary. Objects with $T_{J}>3$ are dynamically decoupled from Jupiter and considered asteroidal. Finally, this dynamical distinction arises from different evolutionary histories: the eccentric orbits of comets reflect their origins in the distant, icy Kuiper Belt and Oort Cloud, while the stable, circular orbits of the main-belt asteroids indicate the occupation of their current locations in the inner solar system since their formation.

These classical definitions are of course of declining usefulness in light of recent research. Observationally, comets do not appear fuzzy at all times. They pass through inactive phases, either on the outer portions of their orbits where the temperatures are too low for sublimation to occur, as a result of mantling, or at the end of their active lifetimes when repeated visits near the Sun have exhausted their volatile supplies (e.g., Hartmann et al. 1987). Even when comets are active, their activity may be so weak as to 
escape notice, further complicating the observational distinction between asteroids and comets.

Physically, asteroids are also known to contain volatile materials, or at least to have contained them in the past. Studies of meteorites traced back to the main asteroid belt have revealed the presence of aqueously altered minerals, indications that liquid water and therefore water ice were at least once present in main belt objects (e.g., Hiroi et al. 1996; Burbine 1998; Keil 2000). Reflectance spectra of the asteroid 1 Ceres have also been found to possess an absorption feature at $3.1 \mu \mathrm{m}$, an indication of possible current surface water ice (Lebofsky et al. 1981; Vernazza et al. 2005).

From a dynamical standpoint, distinguishing comets and asteroids becomes difficult when one takes non-gravitational orbit perturbations into account. Despite being undisputed observationally as a comet, $2 \mathrm{P} /$ Encke occupies an orbit considered by conventional dynamical measures to be non-cometary, with strong gas jet-driven dynamical evolution determined to be the likely reason for this discrepancy (Steel \& Asher; Fernández et al. 2002; Pittich et al. 2004). Likewise, it is possible for main-belt asteroids to be pushed into chaotic resonance zones and subsequently delivered onto Earth-crossing, almost cometlike, orbits via the Yarkovsky effect (Bottke et al. 2002 and references within).

In recent years, many objects have been found to possess characteristics of both asteroids and comets, thus forming a broad new category of so-called comet-asteroid transition objects. Transition objects that have been recently studied include the dynamically asteroidal $\left(T_{J}=3.08\right)$ but observationally cometary 107P/(4015) Wilson-Harrington (Osip et al. 1995; Campins et al. 1995; Chamberlin et al. 1996; Fernández et al. 2005), nearEarth asteroids with comet-like orbits (e.g., Binzel et al. 2004; Fernández et al. 2005), the Damocloids (e.g., Jewitt 2005; Fernández et al. 2005), and asteroids associated with meteor streams (e.g., Babadzhanov 2001; Meng et al. 2004; Williamns et al. 2004; Hsieh \& Jewitt 2005). In this paper, we review a hitherto less-discussed class of transition objects, that of bodies orbiting in the main asteroid belt yet displaying comet-like behavior: active asteroids. The reader is also referred to a recent review of this topic in Comets II (Jewitt 2004).

\section{The Strange Case of $133 \mathrm{P} /(7968)$ Elst-Pizarro}

A discussion of active asteroids necessarily begins with 133P/(7968) Elst-Pizarro (hereafter EP), it being the first and only currently known active main-belt asteroid. An extensive analysis of EP has been presented in Hsieh et al. (2004). The following is a summary of the main points presented in that paper.

Previously identified as an inactive asteroid $\left(1979 \mathrm{OW}_{7}\right)$, EP's active nature became apparent in 1996 when a dust trail was observed by Eric Elst and Guido Pizarro (Elst et al. 1996). Numerical modeling by Boehnhardt et al. (1996) indicated that the dust trail was formed from dust emitted over several weeks or months, a finding inconsistent with a single instantaneous impact event and strongly suggestive of cometary (sublimationdriven) activity. Given the unexpectedness of such activity, however, considering EP's apparently stable orbit in the main asteroid belt where no other comet-like bodies had ever been observed, controversy remained over the true cause of EP's activity, whether cometary (e.g., Boehnhardt et al. 1998) or impact-driven (e.g., Tóth 2000).

Observations in 2002 showing that EP's dust trail had returned, however, eliminated the possibility that impacts alone could be responsible. In Hsieh et al. (2004), we reported observations of EP from four occasions in 2002 (August, September, November, and December) during which the trail was visible (Figure 1). The recurrence of the trail is itself a strong argument for a sublimation-driven origin due to the implausibility of two 


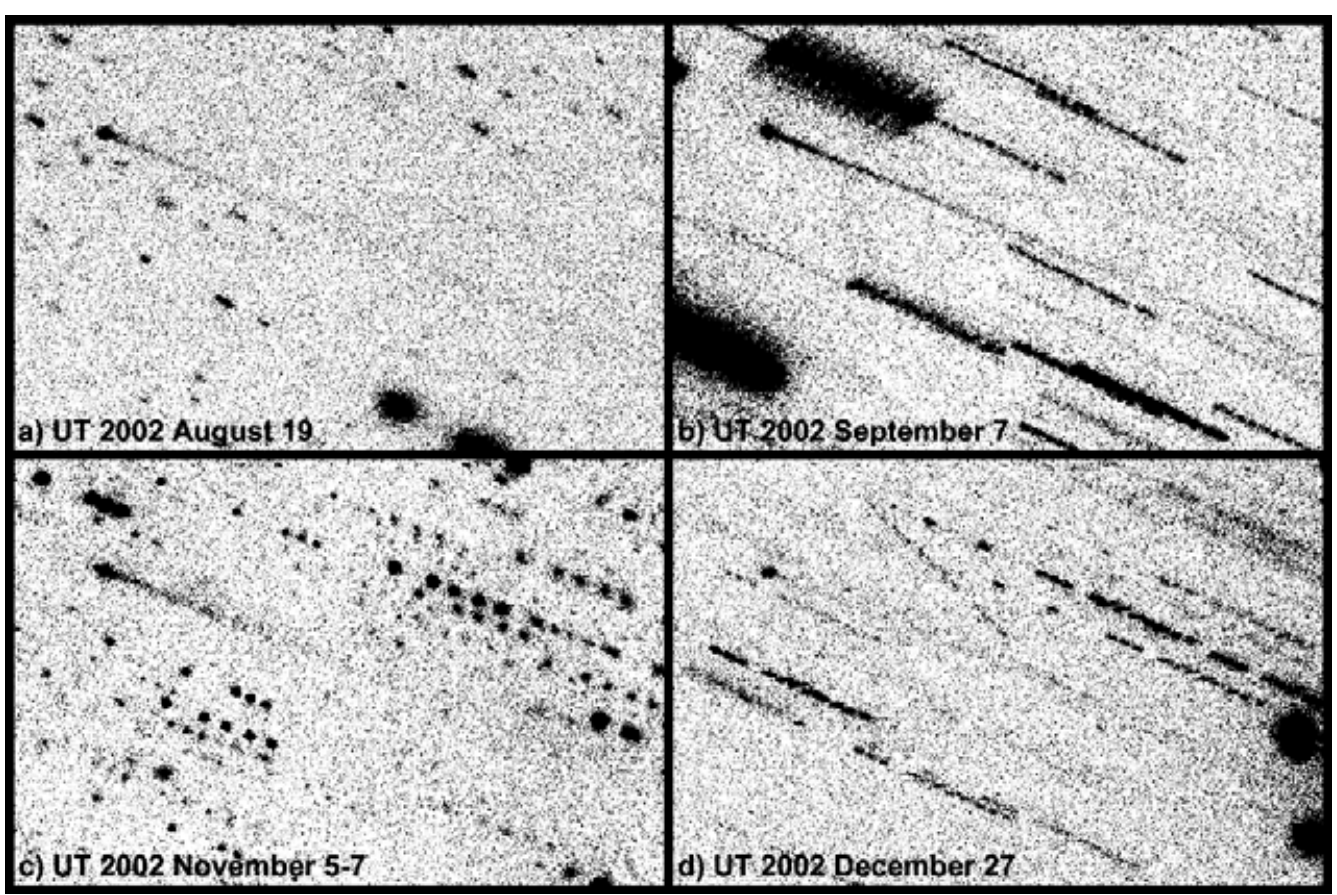

Figure 1. Composite R-band images of 133P/Elst-Pizarro from observations on the University of Hawaii $2.2 \mathrm{~m}$ telescope. Images represent 2500, 3900, 4500, and $4200 \mathrm{~s}$ in effective exposure time, respectively, and are 2 arcmin by 1.5 arcmin in size, with North at the top and East to the left. The nucleus is located at the same position in each image in the upper left corner with the dust trail extending down and to the right. Shorter, dotted trails and other point-like sources are background stars, galaxies, and field asteroids trailed due to the non-sidereal motion of the telescope in tracking the object. Image from Hsieh et al. (2004).

impact events on the same object over the span of 6 years, while no other similar events have been observed on any other main belt object in the entire history of observations of this population. The longevity of EP's dust trail lends further support for a sublimationdriven explanation of EP's activity, since ejecta from a simple impact would not linger for the months that EP's trail was observed to persist in both 1996 and 2002 and then rapidly dissipate in a matter of weeks, as observed in 2002.

In Hsieh et al. (2004), we also presented detailed results of Finson-Probstein modeling in which we found that no single impulsive emission model (equivalent to an impact event) could fully account for the behavior of EP's trail (its persistence over months, great length with no observable detachment from the nucleus, and eventual rapid disappearance over weeks). The incompatibility of impulsive emission models and actual observations of EP is exemplified by Figure 2, in which linear surface brightness profiles of modeled dust tails due to impulsive emission are seen to be clearly poor fits to the linear surface brightness profile of EP's dust trail as observed in 2002 September. In contrast, a continuous emission model (approximated as a superposition of multiple, consecutive impulsive emission models like those in Figure 2) can be seen in Figure 3 to be far superior in matching EP's observed surface brightness profile. The much closer fit of this model to the data emphasizes our previous conclusion: EP's trail must have been produced by extended dust emission episode, a scenario consistent with sublimation-driven, i.e. cometary, activity. 


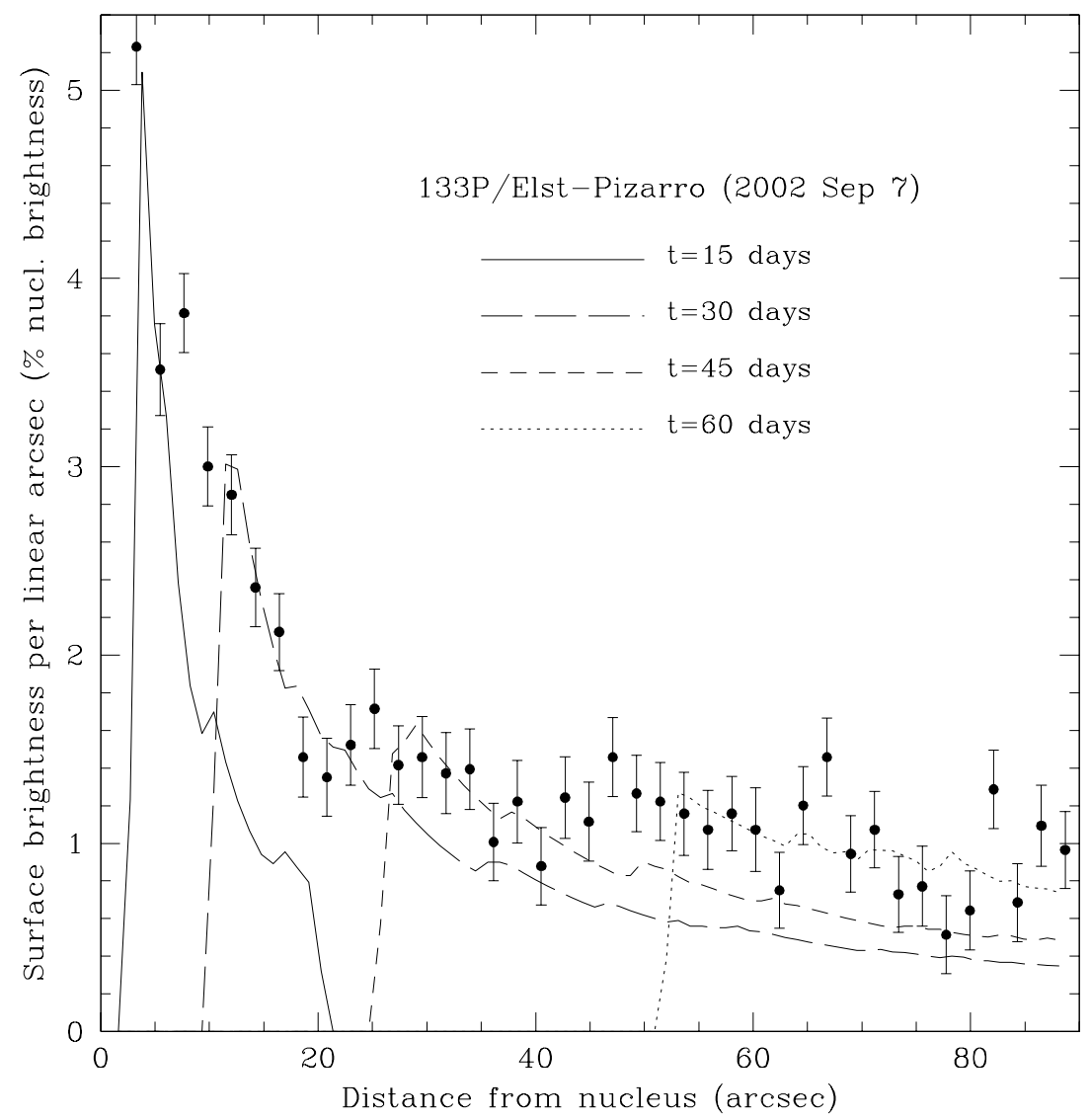

Figure 2. Sample fit of superposed profiles of Finson-Probstein model trails generated from an impulsive emission model to observed data points from 2002 Sep 7 , where $t$ is the time of emission in terms of the number of days prior the observation period, and particle sizes and ejection velocities are held constant. For an average nucleus $R$-band magnitude of 19.7 from this observing period, one trail surface brightness unit is equivalent to $24.7 \mathrm{mag}$ per linear arcsec. Model profiles are arbitrarily scaled to approximate observed data. Image from Hsieh et al. (2004).

\subsection{Activity Modulation}

Ordinarily, the onset of sublimation-driven activity in a comet corresponds with its transition from the outer solar system (roughly beyond the orbit of Jupiter) into the inner solar system along its highly eccentric orbit. EP's orbit in the main asteroid belt, however, is entirely confined to the inner solar system (ranging from 3.7 AU at aphelion to 2.6 AU at perihelion), indicating that another modulation mechanism may be required. The 6 year interval between observations of EP's dust trail in 1996 and 2002 corresponds closely to EP's 5.6 year orbit period, suggesting that its activity could be seasonally modulated. The geometry that would give rise to such a scenario is illustrated in Figure 4, where EP's obliquity is non-zero and exposed volatile material is confined to a single isolated patch near one of the nucleus's rotational poles. 


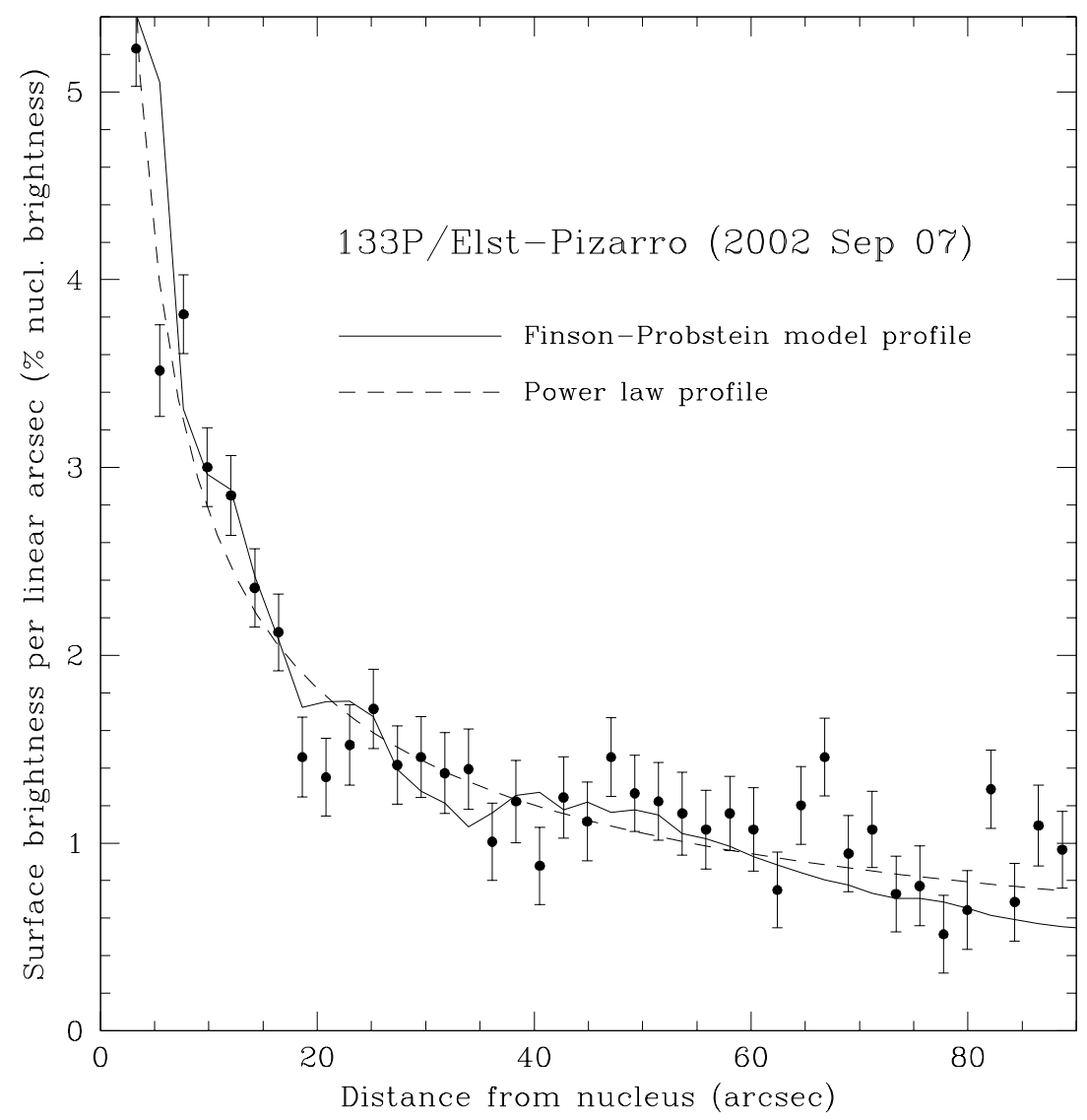

Figure 3. Sample fit of profile of single Finson-Probstein model trail generated from $12 \mathrm{im}-$ pulsive events of varying intensity over 60 days prior to the observation period, intended to simulate continuous emission, to observed data points from 2002 Sep 7. Particle sizes and ejection velocities are held constant. Also shown for reference is a power-law profile, $\gamma^{-0.6}$, where $\gamma$ is projected distance from the nucleus in arcsec. For an average nucleus $R$-band magnitude of 19.7 from this observing period, one trail surface brightness unit is equivalent to 24.7 mag per linear arcsec. The model profile is arbitrarily scaled to approximate observed data. Image from Hsieh et al. (2004).

As shown in the diagram, during the volatile hemisphere's "winter," when its pole points away from the Sun, the volatile patch receives little solar heating and thus does not sublimate appreciably, causing EP to appear inactive. During the active hemisphere's "summer," however, the volatile patch receives much greater exposure to the Sun and thus begins to sublimate, ejecting dust particles as it does, producing EP's observed dust trail. This hypothesis is consistent with current observations, in which EP is only seen to be active while in the quadrant of its orbit immediately after its perihelion passage, and will soon permit a simple observational test following EP's next perihelion passage on 2007 July 1. If the seasonal heating hypothesis holds true, a dust trail should develop again near this time and persist until late 2008. Recurrence of dust emission prior to this time, while not ruling out the seasonal heating hypothesis, would imply the presence of multiple active sites, a conclusion which would then have implications for the possible 


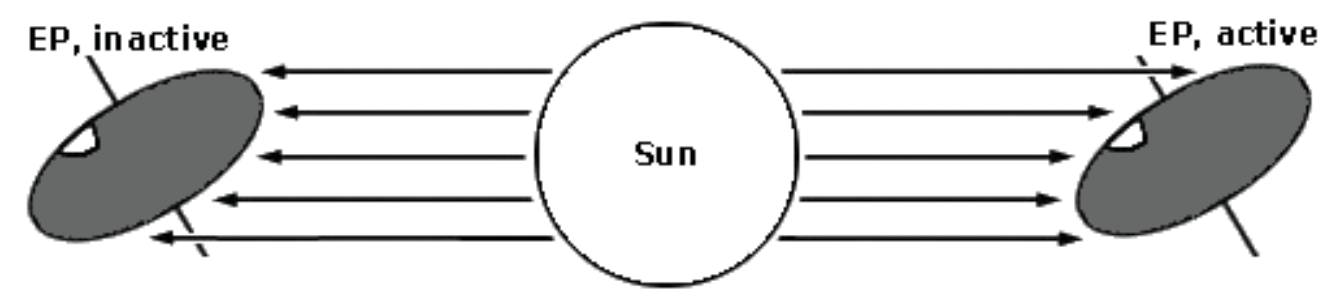

Figure 4. Schematic diagram illustrating seasonal heating of an isolated volatile patch on EP's surface. During EP's inactive phase, its rotational obliquity prevents an isolated patch of volatiles (shown in white) from receiving enough solar radiation to sublimate. During EP's active phase, the volatile patch becomes fully exposed to the Sun (during that hemisphere's "summer"), is heated, and ejects surface material as it sublimates, generating a visible dust trail.

origin of these active sites, as we will discuss in the following section. The non-recurrence of dust emission, however, would effectively exclude the seasonal heating hypothesis and additionally cast doubt on the sublimation hypothesis itself.

\section{Explaining Elst-Pizarro}

Given this surprising find of a dynamically asteroidal object outgassing like a comet, we must now ask how such a strange object is possible. We consider two hypotheses: either EP is a barely active Jupiter-family comet (JFC) that perhaps has evolved onto its current orbit via the long-term non-gravitational influence of asymmetrical cometary outgassing, or (2) EP is a true native member of the asteroid belt on which preserved, buried ice has been excavated by a recent impact, i.e. an activated asteroid. (For clarity, for the remainder of this paper, we will use the term "activated asteroid" to specifically refer to objects described by this latter hypothesis, while "active asteroid" will be used to refer to any currently dynamically asteroidal object displaying cometary behavior without regard to the origin of the body or the activity.)

\subsection{A Lost Comet?}

If EP is an ordinary JFC that has somehow evolved onto an asteroidal orbit, the fact that it contains volatiles and is currently outgassing is easily explained. Its orbit, however, is not. With $T_{J}=3.18$, EP is completely decoupled from Jupiter, unlike most other JFCs. Such a decoupling may have come about through a combination of perturbations from close encounters with the terrestrial planets or the influence of other non-gravitational forces, such as asymmetrical cometary outgassing. EP's activity is presently extremely weak, and at its current strength, is unlikely to be able to significantly affect its orbit. It should be noted, however, that earlier in its life, EP probably contained significantly larger quantities of volatile material and thus may have had much stronger outgassing that could then have played a much larger role in EP's dynamical behavior and evolution.

Ipatov \& Hahn (1997) found EP's current orbit to be quite stable under purely gravitational influences, meaning that the evolution of a JFC onto such an orbit under those same influences would be unlikely. Fernández et al. (2002) also attempted to model the dynamical evolution of a JFC (D/Pigott) onto an EP-like orbit under gravitational influences but could not reproduce EP's low inclination. The inclusion of non-gravitational forces, i.e., cometary outgassing, could significantly change the results of these models, however, and for this reason, we cannot rule out the possibility that EP is a lost comet.

It should also be noted that Comet $2 \mathrm{P} /$ Encke also possesses a purportedly asteroidal Tisserand invariant $\left(T_{J}=3.03\right)$ though its identification as a comet is not in question. 
Non-gravitational forces are thought to have played and be currently playing a large role in Encke's dynamical evolution (Steel \& Asher; Fernández et al. 2002; Pittich et al. 2004). Clearly, comets are capable of occupying canonically non-cometary orbits and likely move onto such orbits under the influence of non-gravitational cometary outgassing. That being said, EP certainly has the "most" asteroidal orbit of any known comet, sitting precisely amid one of the most populous asteroid families in the main belt. The evolution of a JFC onto such an orbit would certainly be an extraordinary coincidence, but given EP's current observational uniqueness, cannot be completely ruled out.

\subsection{Or an Icy Asteroid?}

The idea that ordinary main belt asteroids might contain ice is not a new one. Spectral features attributed to water of hydration have been observed for main-belt asteroids in the infrared at $3 \mu \mathrm{m}$ (e.g., Lebofsky 1980; Lebofsky et al. 1981; Feierberg et al. 1985; Jones et al. 1990; Hasegawa et al. 2003) and in the visible at $0.7 \mu \mathrm{m}$ (e.g., Vilas et al. 1994; Barucci et al. 1998; review by Rivkin et al. 2002). The primordial presence of water ice and liquid water in the main belt has likewise been inferred by the presence of hydrated minerals in meteorites, specifically aqueously altered CI and CM carbonaceous chondrites, found here on Earth and determined to have originated from the main belt, specifically from C, G, B, and F-type asteroids (e.g., Hiroi et al. 1996; Burbine 1998; Keil 2000). These minerals are generally thought to have formed via the primordial accretion of icy grains into the parent bodies of the carbonaceous chondrites (yr et al. 1998; Mousis \& Alibert 2005) and the subsequent heating (either by the radioactive decay of ${ }^{26} \mathrm{Al}$ or electromagnetic induction from the solar wind) and liquification of that water ice, leading to aqueous alteration of those parent bodies (Grimm \& McSween 1989; Cohen \& Coker 2000; Rosenberg et al. 2001).

Given the apparent ubiquity of primordial water implied by spectroscopic and meteoritic evidence, Jones et al. (1990)] then argued that a decline in detections of hydrated silicates in asteroids of increasing semimajor axis in the main belt could simply be indicative of declining heating effects with increasing distance from the Sun. The absence of hydration features would therefore be because the ice in more distant objects was never heated to the liquid state and thus no hydration reactions were possible. That ice could then still exist today. A similar conclusion was reached by Scott \& Krot (2005) who argued that the existence of pristine, unaltered carbonaceous chondrites indicated not that the parent bodies of those chondrites had actually been ice-free, but had instead simply escaped significant heating and could therefore still contain significant quantities of preserved water ice at the present day.

Thermal models appear to support such conclusions. Indeed, even when alteration has occurred, modeling by Grimm \& McSween (1989) of CM chondrite production predicts that for small parent bodies, the bulk of each body's ice must remain frozen in order to produce the temperature distribution dictated by the aqueous alteration models. They additionally suggest that ice in large asteroids, e.g., Ceres, at 3 AU that survived primordial heating could be stable against sublimation from ordinary solar heating at depths of only tens of meters, a result corroborated by modeling by Fanale \& Salvail (1989).

In fact, water ice has actually been detected for Ceres. Lebofsky et al. (1981) reported an absorption feature at $3.1 \mu \mathrm{m}$ in a near-infrared reflectance spectrum of the body, indicating possible water ice, perhaps in the form of a "surface frost." This detection was later bolstered by A'Hearn \& Feldman (1992), who reported detecting OH vapor from Ceres that could have been photodissociated $\mathrm{H}_{2} \mathrm{O}$ evaporating from a seasonallyvarying northern polar ice cap, and Vernazza et al. (2005), who confirmed the existence of absorption at $3.06 \mu \mathrm{m}$, finding it to be consistent with a mix of crystalline water ice 
and residues of ion-irradiated asphaltite. Thus the question of whether water ice can exist in the main belt, at least on a body as large as Ceres, has already been answered. With the discovery and confirmation of EP's comet-like activity, the present-day existence of water ice on much smaller asteroids is now also established.

\section{Why Haven't Other Active Asteroids Been Seen?}

The previous discussion brings up a natural question: if water ice is thought to be prevalent in outer belt asteroids, why hasn't outgassing been observed in other asteroids besides EP? Perhaps the most straightforward explanation may be that no one has yet looked closely enough at enough asteroids to notice the weak, transient, EP-like activity that may be typical of active asteroids. While many larger asteroids have been studied in detail, EP $\left(r_{\text {eff }} \sim 2.5 \mathrm{~km}\right.$; Hsieh et al. 2004) falls in the far more populous group of small main-belt asteroids that are generally not imaged deeply enough in either discovery or recovery images that are taken for astrometric purposes only, and then subsequently do not attract significant follow-up study due to their small sizes (and thus faint apparent magnitudes) and ordinary dynamical properties. In August 2002, EP's trail was just barely visually noticeable in a 5-minute, R-band image taken with the UH $2.2 \mathrm{~m}$ telescope on Mauna Kea, indicating that comparable activity from other asteroids may only be detectable by imaging potentially active asteroids to comparable depths from comparably-sized telescopes under comparably good observing conditions.

Observational biases aside, not every small asteroid is expected to be active, of course. Under the activated, icy asteroid hypothesis, a recent collision is also necessary for the creation of an activated asteroid, and presuming the struck asteroid is actually icy (which of course is not guaranteed), this collision must specifically strike an icy portion of that asteroid. If subsurface ice is non-uniformly distributed (not an implausible scenario given likely uneven solar heating effects due to particular rotational axis orientations or local non-uniformities in composition throughout an asteroid), not every impact onto an icy asteroid would necessarily constitute an activation. Even the successful activation of an asteroid, however, does not then guarantee the detection of the resulting activity. In addition to possibly being quite weak, as discussed above, the activity of an activated asteroid is expected to be intermittent, requiring observations to be made at the right time. In principle, this means that even inactive objects would need to be continuously monitored in order to ensure that any outbursts are not missed. Furthermore, in analogy to the comets, active sites on activated asteroids likely have finite lifetimes, limited by either actual total local devolatilization of the active site or mantling (Jewitt 1996 and references within). Thus, asteroids that may have been active in the recent past may not necessarily show current activity, much as past activity is inferred for asteroidal parents of meteor streams, such as 3200 Phaethon (parent of the Geminids), despite the absence of any currently observed activity (e.g., Hsieh \& Jewitt 2005).

In addition to all the above considerations, the creation and detection of activated asteroids may be even further complicated by a conflict in size preferences that suggests that detectable activated asteroids may only occupy a very narrow range of sizes. On the one hand, the rate of impact excavations of a particular asteroid is dependent on the collisional cross-section of a body. Larger bodies are expected to be struck more often. Thus, it may appear that larger asteroids are more likely to become activated and therefore should form the focus of any observational search for additional activated asteroids. Larger asteroids are also better suited for preserving volatiles from solar heating over large timescales simply because those volatiles can be buried at greater depths than on smaller asteroids, and become exposed later via a particularly deep collisional 
excavation, collisional disruption of the parent body (either complete fragmentation of the body or the shearing-off of large surface fragments), or gradual "percolation" to the surface.

On the other hand, another key issue may be the extremely low dust ejection velocity $\left(\sim 1-2 \mathrm{~m} \mathrm{~s}^{-1}\right)$ we found for EP, comparable to the gravitational escape velocity $\left(v_{e s c} \sim\right.$ $1 \mathrm{~m} \mathrm{~s}^{-1}$ ) of the roughly 5 -km body. Dust emission on larger bodies may never actually become observable due to the larger escape velocities on those bodies. Volatile material could be sublimating but the gas drag forces generated might simply be too weak to eject dust particles into an observable dust coma or trail. For example, this may be why no dust emission has ever been observed for Ceres, despite the detection of water vaporization by A'Hearn \& Feldman (1992). Ejected dust from smaller bodies would be more likely to be able to escape, but those smaller bodies also present smaller collisional cross-sections per object and would not experience as high a rate of activations in the first place as larger bodies. Smaller bodies are also less able to insulate interior ices from long-term solar heating, as discussed above, but are also less effectively heated from within by ${ }^{26} \mathrm{Al}$ (since smaller objects have a greater surface area per unit volume and thus radiate the heat generated by ${ }^{26} \mathrm{Al}$ decay more efficiently than larger objects) and so may therefore be less altered. This conflict in size preferences suggests that activated asteroids may be even rarer and more difficult to discover than initial considerations might indicate.

Finally, we note that at the present time, we still cannot eliminate the possibility that $\mathrm{EP}$ is a bona fide comet that has managed to stray into the main belt. If this is the case, the expected rarity of this dynamical transition would suggest that EP may be unique, precluding the existence of any other active asteroids in the main belt, explaining why none other than EP have yet been observed.

\section{Finding Other Active Asteroids}

If EP is an icy asteroid, other EP-like objects should exist and should be found. The question then is how to go about looking for them. An issue of likely importance is EP's location in the Themis collisional family. This family is roughly defined by orbital element ranges $3.047 \mathrm{AU}<a<3.220 \mathrm{AU}, 0.119<e<0.191$, and $0.688^{\circ}<\sin i<2.235^{\circ}$, where $a$ is semimajor axis, $e$ is eccentricity, and $i$ is inclination (Zappalá et al. 1990), neatly bracketing the orbital elements of $\operatorname{EP}\left(a=3.156 \mathrm{AU}, e=0.165, \sin i=1.386^{\circ}\right)$. As the result of the catastrophic disruption of a large parent body, perhaps hundreds of $\mathrm{km}$ in size (Marzari et al. 1995), the Themis family is thought to consist of related asteroids of similar composition. This conclusion has been supported thus far by studies (e.g., Florczak et al. 1999; Ivezić et al. 2002) of asteroid families that find approximate spectral homogeneity among members, finding the Themis family in particular to be dominated by C-type asteroids, which, again, are considered to be one of the dominant sources of aqueously altered CM and CI meteorites. Thus, in the search for objects that are behaviorally similar (and therefore probably compositionally similar) to EP, assuming $\mathrm{EP}$ is not an interloper of cometary origin or from elsewhere in the main belt, the rest of the Themis family is a logical place to start.

The nature of families as being formed from the catastrophic disruption of larger parent bodies also suggests the prospect that these much larger parent bodies may have protected large quantities of interior ices against early heating episodes, such as a possible early solar wind induction heating episode (e.g., Jones et al. 1990), as well as ordinary solar heating over the billions of years since the birth of the solar system. For example, the Themis family is estimated to have been formed perhaps hundreds of Myr to 2 Gyr ago from the disruption of a parent body hundreds of kilometers in size (Marzari et al. 
1995). Ice inside the Themis parent body could therefore have been preserved for the first few billion years of the life of the solar system and then distributed among smaller bodies, of which EP may be one, upon the destruction of the parent body.

The possible need for collisional activation of EP is also consistent with its location among the Themis family, the family being characterized by a higher than typical collision rate due to the large numbers of asteroids being clustered in orbital element space (Farinella \& Davis 1992; Dell'Oro et al. 2001). This then suggests that the Koronis family, characterized by even higher collision probabilities than the Themis family (Farinella \& Davis 1992), could be a similarly promising region to survey for other activated asteroids. Like the Themis family, the Koronis family appears to be the result of the catastrophic fragmentation of a large parent asteroid about 2 Gyr ago (Marzari et al. 1995), and so could likewise contain ice that was deeply buried and preserved for billions of years within the Koronis family parent body, but that now resides in much smaller asteroids following the fragmentation of that parent body. Unlike the Themis family, the Koronis family is dominated by S-type asteroids (Bell 1989; Binzel et al. 1993; Mothé-Diniz et al. $2005)$ which along with their analogs, the ordinary chondrites, are not known to show significant aqueous alteration (e.g., Rivkin et al. 2002) and thus may be less likely to contain reserves of water ice that might be able to become activated. Controversy exists over the purported anhydrousness of the ordinary chondrites (e.g., Grossman et al. 2000, Keil 2000), however, and so for this reason, it is not possible to entirely rule out the Koronis family as a potential enclave of activated asteroids.

The recent identification and age determination of extremely young families, namely the Veritas family (8.3 Myr) and Karin and Iannini clusters (5.8 Myr and <5 Myr, respectively; Nesvorný et al. 2002; Nesvorný et al. 2003), suggest that these too could be fruitful regions to search for activated asteroids. The extremely young ages of these families suggests that any ices buried deeply within the interiors of the families' parent bodies would only have become exposed upon the disruption of those parent bodies and formation of the families in the last few million years. That ice could then very reasonably still persist on or near the surfaces of the resulting fragments at the present day, and thus be able to drive EP-like, sublimation-driven dust emission. Being young, these families are also still quite tightly clustered in orbital element space, indicating a probable enhanced probability of intrafamily collisional activations in the event that ices were not exposed outright by the initial fragmentation of the families' parent bodies. As before, asteroid types may indicate which of these young families is most likely to exhibit activity - the C-type Veritas family appearing to be a more promising search region than the S-type Karin or Iannini clusters - but uncertainty in the role of taxonomic types in indicating true water content means that none of these families should yet be completely dropped from consideration as possible reservoirs of activated asteroids.

\section{Challenges and Future Work}

Foremost among the challenges we face in the study of active asteroids is, of course, the fact that only one is known: EP. It is therefore difficult to conclusively identify which properties of EP are most significant in its production of comet-like dust emission and which observational signatures would be most usefully exploited in the search for EP analogs. For example, we discussed above the need for caution in using taxonomic types to definitively declare which asteroids should be expected to display activity and which should not. The possible significance of EP's particular size was also discussed earlier. The unusually rapid rotation of EP $\left(P_{\text {rot }}=3.471 \mathrm{hr}\right.$; Hsieh et al. 2004) may also play an important role in its cometary behavior by imparting centrifugal force to gas-ejected 
dust, aiding its escape from the surface of the body into the dust trail. Interestingly, another comet-asteroid transition object, the observationally inactive 3200 Phaethon that has nonetheless been associated with the Geminid meteor stream (Whipple 1983; Gustafson 1989; Williamns \& Wu 1993), implying past comet-like dust emission, also rotates quite rapidly $\left(P_{\text {rot }}=3.60 \mathrm{hr}\right.$; Krugly et al. 2002). Whether EP-like dust ejection is equally likely on slower rotators, however, is difficult to say without knowing the range of strengths of asteroidal ice sublimation, information which necessarily requires a larger sample (more than one, at least) of known active asteroids to constrain.

Another major obstacle in assessing EP's nature is the difficulty of confirming or rejecting the possibility that EP is a highly-evolved JFC. The intuitive sentiment that such a situation, if true, should be so rare that EP must be unique is currently supported by the available observational evidence. Without a good statistical understanding of exactly how rare this situation should be, however, this hypothesis is difficult to conclusively observationally assess (particularly since the statistical likelihood of the competing hypothesis that EP is an activated asteroid is also considered low but is currently likewise poorly constrained). More detailed dynamical evolution models of JFCs, with particular attention paid to the role of non-gravitational perturbations exerted by cometary outgassing, to determine whether the transition of a JFC onto a main-belt orbit (and into the midst of the Themis family in particular) is actually impossible, merely nearly impossible, or perhaps not as difficult as currently thought, would therefore obviously be quite valuable in helping to reveal EP's true nature and determining whether we should actually expect to find any other EP-like objects in the main belt.

In any event, it is clear that an effective examination of EP and the nature of its activity is impossible without accompanying campaigns to discover other EP-like objects or ruling out their existence. This can (and is) being done at the current time in pointed surveys of selected small main-belt asteroids such as the Hawaii Trails survey project we have been conducting at the University of Hawaii, but the sheer number of possible targets (numbering in the thousands) that satisfy the size and family-association criteria described above means that such campaigns will eventually be much better served by large-scale synoptic surveys, such as the University of Hawaii's Pan-STARRS, soon to come online. In the meantime, the discovery of even one more active asteroid in the main belt either by the Hawaii Trails project or a similar survey, or by serendipitous discovery, would obviously be quite a significant step forward, permitting the refinement of target selection criteria, thus focusing the scope of future survey campaigns and greatly facilitating the discovery of even more active asteroids and furthering our understanding of these mysterious objects.

\section{Acknowledgements}

We thank Humberto Campins for helpful comments on this manuscript. This work was supported in part by a grant to DJ from NASA and a travel grant to HHH from the International Astronomical Union.

\section{References}

A'Hearn, M.F. \& Feldman, P.D. 1992, Icarus 98, 54

Babadzhanov, P.B. 2001, A\& A, 373, 329

Barucci, M.A., Doressoundiram, A., Fulchignoni, M., Florczak, M., Lazzarin, M., Angeli, C., \& Lazzaro, D. 1998, Icarus 132, 388

Bell, J.F. 1989, Icarus, 78, 426

Binzel, R.P., Xu, S., \& Bus, S.J. 1993, Icarus, 106, 608 
Binzel, R.P., Rivkin, A.S., Stuart, J.S., Harris, A.W., Bus, S.J., \& Burbine, T.H. 2004, Icarus, 170,259

Boehnhardt, H., Schulz, R., Tozzi, G.P., Rauer, H., \& Sekanina, Z. 1996, IAUC 6495

Boehnhardt, H., Sekanina, Z., Fiedler, A., Rauer, H., Schulz, R., \& Tozzi, G. 1998, Highlights in Astronomy 11, 233

Bottke, W.F., Vokrouhlický, D., Rubincam, D.P., \& Broz, M. 2002, in: W.F. Bottke Jr., A. Cellino, P. Paolicchi \& R.P. Binzel (eds.), Asteroids III (Tucson: University of Arizona Press), p 395

Burbine, T.H. 1998, Meteoritics \& Planetary Science, 33, 253

Campins, H., Osip, D. J., Rieke, G. H., \& Rieke, M. J. 1995, Planet. Es Space Sci., 43, 733

Chamberlin, A.B., McFadden, L.-A., Schulz, R., Schleicher, D.G., \& Bus, S.J. 1996, Icarus, 119, 173

Cohen, B. A. \& Coker, R. F. 2000, Icarus, 145, 369

Cyr, K.E., Sears, W.D., \& Lunine, J.I. 1998, Icarus 135, 537

Dell'Oro, A., Paolicchi, P., Cellino, A., Zappalà, V., Tanga, P., \& Michel, P. 2001, Icarus, 153, 52

Elst, E. W., Pizarro, O., Pollas, C., Ticha, J., Tichy, M., Moravec, Z., Offutt, W., \& Marsden, B.G. 1996, IAUC 6496

Fanale, F.P. \& Salvail, J.R. 1989, Icarus, 82, 97

Farinella, P. \& Davis, D.R. 1992, Icarus, 97, 111

Feierberg, M. A., Lebofsky, L. A., \& Tholen, D. J. 1985, Icarus, 63, 183

Fernández, Y.R., McFadden, L.A., Lisse, C.M., Helin, E.F., \& Chamberlin, A.B. 1997, Icarus, 128,114

Fernández, J.A., Gallardo, T., \& Brunini, A. 2002, Icarus, 159, 358

Fernández, Y.R., Jewitt, D.C., \& Sheppard, S.S. 2005, AJ, 130, 308

Florczak, M., Lazzaro, D., Mothé-Diniz, T., Angeli, C.A., \& Betzler, A. S. 1999, A $\& A S, 134$, 463

Grimm, R.E. \& McSween, H.Y., Jr. 1989, Icarus, 82, 244

Grossman, J.N., Alexander, C.M.O., Wang, J., \& Brearley, A.J. 2000, Meteoritics and Planetary Science, 35, 467

Gustafson, B. Å. S. 1989, A\&̈A, 225, 533

Hartmann, W.K., Tholen, D.J., \& Cruikshank, D.P. 1987, Icarus, 69, 33

Hasegawa, S., Murakawa, K., Ishiguro, M., Nonaka, H., Takato, N., Davis, C.J., Ueno, M., \& Hiroi, T. 2003, GeoRL, 30, 2

Hiroi, T., Zolensky, M.E., Pieters, C.M., \& Lipschutz, M.E. 1996, MESPS, 31, 321

Hsieh, H.H., Jewitt, D.C., \& Fernández, Y.R. 2004, AJ, 127, 2997

Hsieh, H. H. \& Jewitt, D. 2005, ApJ, 624, 1093

Ipatov, S. I. \& Hahn, G. J. 1997, in: Lunar and Planetary Science XXVIII (Houston: Lunar Planet. Inst.), 619

Ivezić, Ž., Lupton, R.H., Jurić, M., Tabachnik, S., Quinn, T., Gunn, J.E., Knapp, G.R., Rockosi, C.M., \& Brinkmann, J. 2002, AJ, 124, 2943

Jewitt, D. 1996, Earth, Moon and Planets, 72, 185

Jewitt, D. 2004, in: M.C. Festou, H.U. Keller \& H.A. Weaver (eds.), Comets II (Tucson: University of Arizona Press), p 659

Jewitt, D. 2005, AJ, 129, 530

Jones, T.D., Lebofsky, L.A., Lewis, J.S., \& Marley M.S. 1990, Icarus, 88, 172

Keil, K. 2000, Planet. \& Space Sci., 48, 887

Krugly, Yu. N., Belskaya, I.N., Shevchenko, V.G., Chiorny, V.G., Velichko, F.P., Mottola, S., Erikson, A., Hahn, G., Nathues, A., Neukum, G., Gaftonyuk, N.M., \& Dotto, E. 2002, Icarus, 158, 294

Lebofsky, L. A. 1980, AJ, 85, 573

Lebofsky, L.A., Feierberg, M.A., Tokunaga, A.T., Larson, H.P., \& Johnson, J.R. 1981, Icarus, 48,453

Marzari, F., Davis, D., \& Vanzani, V. 1995, Icarus, 113, 168 
Meng, H., Zhu, J., Gong, X., Li, Y., Yang, B., Gao, J., Guan, M., Fan, Y., \& Xia, D. 2004, Icarus, 169, 385

Mothé-Diniz, T., Roig, F., \& Carvano, J. M. 2005, Icarus, 174, 54

Mousis, O. \& Alibert, Y. 2005, MNRAS 358, 188

Nesvorný, D., Bottke, W.F., Dones, L., \& Levison, H.F. 2002, Nature, 417, 720

Nesvorný, D., Bottke, W.F., Levison, H.F., \& Dones, L. 2003, ApJ, 591, 486

Osip, D., Campins, H., \& Schleicher, D.G. 1995, Icarus, 114, 423

Pittich, E.M., D'Abramo, G., \& Valsecchi, G.B. 2004, A\&A, 422, 369

Rivkin, A.S., Howell, E.S., Vilas, F., \& Lebofsky, L.A. 2002, in: W. F. Bottke Jr., A. Cellino, P. Paolicchi, \& R. P. Binzel (eds.), Asteroids III (Tucson: University of Arizona Press), p 235

Rosenberg, N.D., Browning, L., \& Bourcier, W.L. 2001, MEPS, 36, 239

Scott, E.R.D. \& Krot, A.N. 2005, ApJ, 623, 571

Steel, D.I. \& Asher, D.J. 1996, MNRAS, 281, 937

Tóth, I. 2000, $A \mathscr{G} A, 360,375$

Vernazza, P., Mothé-Diniz, T., Barucci, M.A., Birlan, M., Carvano, J.M., Strazzulla, G., Fulchignoni, M., \& Migliorini, A. 2005, A\& $A, 436,1113$

Vilas, F., Jarvis, K.S., \& Gaffey, M.J. 1994, Icarus 109, 274

Whipple, F. L. 1983, IAUC 3881

Williams, I.P. \& Wu, Z. 1993, MNRAS, 262, 231

Williams, I.P., Ryabova, G.O., Baturin, A.P., \& Chernitsov, A.M. 2004, MNRAS, 355, 1171

Zappalá, V., Cellino, A., Farinella, P., \& Knežević, Z. 1990, AJ, 100, 2030 